\section{LA EDUCACIÓN SUPERIOR EN TRÁNSITO: ¿ES BOLONIA LA RUTA IDÓNEA PARA LA INNOVACIÓN?}

\author{
Alfonso Unceta Satrústegui \\ Profesor Titular de Sociología \\ UPV/EHU \\ alfonso.unceta@ehu.es
}

\section{HIGHER EDUCATION IN TRANSIT: IS BOLOGNIA THE IDEAL ROUTE FOR INNOVATION?}

\begin{abstract}
The European university is currently living times of change. A change intended to be innovative and whose goal is to create a European Area of Higher Education. This transformation known as the "Bologna Process" commenced at the end of the $20^{\text {th }}$ century and it has consolidated over the last decade. In the following lines, reflections are made on the innovative potentiality of Bologna regarding its practical application in Spain.
\end{abstract}

KEY WORDS: High Education; Bolonia; innovation; change.

\section{INTRODUCCIÓN}

Frente a la creencia popular de que las universidades son instituciones que apenas han variado su sentido y su forma de hacer durante siglos, la realidad da cuenta de un proceso lento pero constante de adaptación, una metamorfosis que ha sido el resultado de cambiantes necesidades sociales, culturales, políticas o económicas. Por hacer referencia sólo al tiempo moderno y contemporáneo, mientras la universidad del siglo XIX está fundamentalmente imbuida por el espíritu y los objetivos de la ilustración y centra su actividad en la formación de las elites, durante el siglo XX la institución universitaria ha proporcionado a millones de ciudadanos la formación especializada que la sociedad industrial requería, y en los comienzos del siglo XXI la demanda social hacia la universidad plantea los desafíos propios de una sociedad atravesada por un intenso y acelerado desarrollo del conocimiento que se encuentra a su vez organizado e interconectado en redes de carácter global ${ }^{1}$.

Estas diferentes maneras de considerar la misión y la acción universitaria han inducido numerosos ajustes y reformulaciones en el ámbito de la educación superior, adaptaciones
RESUMEN: La universidad europea vive tiempos de cambio. Un cambio que se pretende innovador y persigue construir el Espacio Europeo de Educación Superior. Esta transformación, que ha sido conocida como el "Proceso de Bolonia", tiene su origen a finales de los años noventa del pasado siglo y ha ido consolidándose a lo largo de la última década. En las líneas que siguen se reflexiona acerca de las potencialidades innovadoras de Bolonia centrando el análisis en la aplicación práctica en el caso español.

PALABRAS CLAVE: Educación Superior; Bolonia; innovación; cambio.

de orden relativamente menor a distintas influencias y estímulos que, sin embargo, en muchos casos, no han sido suficientemente consideradas. En la actualidad, las universidades encaran el siglo XXI no especialmente atadas a la tradición ni concernidas por ella, sino necesariamente atentas a las grandes innovaciones que caracterizan el tiempo presente. Como señala Brunner, "comienza la historia contemporánea de la universidad; aquella que confronta a estas instituciones con la globalización, con la era de la información, con sociedades que transitan hacia una economía crecientemente basada en la explotación intensa del conocimiento avanzado (y no solo del trabajo humano), con la fragmentación de las comunidades y la individuación de los sujetos; en fin, en el plano cultural, con la postmodernidad o modernidad tardia"2.

Esta aproximación contextual es de gran importancia porque señala la manera en que se conforma y se va comportando la sociedad. Y aunque las características señaladas por Brunner obviamente pueden ampliarse, son de por sí suficientemente ilustrativas del escenario que las universidades han de afrontar para trazar primero y alcanzar después sus objetivos. Atendiendo a dicho escenario y desde la 
óptica educativa, lo que ha venido en llamarse "Proceso de Bolonia" se ha conformado como la ruta que corresponde transitar a las instituciones de Educación Superior en el ámbito europeo.

El "Proceso de Bolonia" tiene una larga historia que empezó a tomar cuerpo, por lo menos de manera parcial, en el año 1988 por medio de la Magna Charta Universitatum suscrita por 388 rectores $^{3}$. En la misma, la apelación a la movilidad y el desarrollo de equivalencias en materia de titulaciones constituyen, en mi opinión, dos pilares fundamentales sobre los que se asentará posteriormente la Declaración de la Sorbona (1998) que lleva por título "Declaración conjunta para la armonización del diseño del Sistema de Educación Superior Europeo"4. Un año más tarde, en 1999, la Declaración de Bolonia ${ }^{5}$ titulada esta vez "El Espacio Europeo de la Enseñanza Superior", recoge de manera explícita los principios sobre los que debe edificarse el Espacio Europeo de Educación Superior. Con posterioridad, distintas ciudades como Praga (2001), Berlín (2003), Bergen (2005), Londres (2007), Lovaina (2009) y Budapest (2010) han sido testigos de sucesivos encuentros y declaraciones de los representantes educativos europeos, que han servido para desarrollar los principios de la Declaración de Bolonia. Tales principios pueden sintetizarse como sigue:

- Establecer un sistema comparable de titulaciones en el ámbito europeo.

- Unificar la estructura de los estudios en dos ciclos: Grado y Postgrado.

- Implantar el sistema de créditos ECTS.

- Impulsar la movilidad estudiantil y docente.

- Promocionar la cooperación europea en materia de aseguramiento de la calidad.

Más de una década ha transcurrido desde la Declaración de Bolonia y en este tiempo han corrido ríos de tinta que han puesto de manifiesto valoraciones y posicionamientos contrapuestos, un panorama en el que se aprecian muchas más discrepancias que unanimidades. Además, como bien señala Fernández Buey, "lo que hay es un contraste notable entre las declaraciones públicas, generalmente eufóricas y optimistas, de la mayoría de las autoridades académicas acerca del Proceso de Bolonia y lo que se oye por abajo, en los pasillos, en los patios y en las reuniones universitarias". . Lo que parece evidente es que Bolonia está lejos de suscitar la adhesión de buena parte de los actores universitarios, aunque lo que pocos discuten son sus implicaciones profundas sobre el funcionamiento de las universidades.

Partiendo de estas premisas, me han interesado especialmente aquellas posiciones fundamentalmente alineadas con la transformación de las prácticas docentes y los procesos de enseñanza/aprendizaje, que definen Bolonia como un proceso innovador que, a su vez, contiene propuestas que son asimismo innovadoras. No tengo dudas de que la educación en general y la universidad en particular son tanto desde el punto de vista analítico como empírico ámbitos propicios para la innovación?

La cuestión que me propongo discutir aquí es hasta qué punto Bolonia pueda considerarse como un proceso innovador y que sus aplicaciones básicas sean realmente innovadoras. Para tratar de dilucidar ambas cuestiones voy a referirme a aspectos tales como la génesis, los objetivos, los actores, y los mecanismos que el "Proceso de Bolonia" incorpora. Un recorrido por los lugares donde Bolonia debe de tomar cuerpo y hacer realidad sus intenciones.

\section{1. ¿ES BOLONIA UN PROCESO INNOVADOR?}

Afirma Echeverría que la innovación puede ser tratada como un objeto de estudio ${ }^{8}$, y recuerda también la importancia de los contextos en orden a favorecer o retardar el desarrollo de procesos de innovación. De hecho, en buena parte de la extensa literatura sobre el concepto de innovación ${ }^{9}$, tienden a subrayarse algunos factores cuya concurrencia favorece el desarrollo de procesos innovadores. Quisiera centrarme en tres de los que parecen fundamentales: la existencia de algún problema, necesidad o demanda que sean necesario encauzar o resolver; la participación de distintos actores y muy especialmente de aquellos más concernidos por el propio proceso innovador; la delimitación de un cierto liderazgo reconocible y aceptado que opera como una referencia del propio proceso. 


\subsection{Problemas que hay que resolver}

Si nos guiamos por los principios que inspiran la Declaración de Bolonia, parecen detectarse tres focos fundamentales de preocupación: la heterogeneidad organizativa (duración de los títulos, contenidos, estructura de los créditos, etc.); la limitada movilidad e intercambio del alumnado y el profesorado; y la escasa penetración de la cultura de la evaluación y la calidad en la universidad.

Sin embargo, y por lo que se refiere a la universidad española, no puede afirmarse que ninguna de estas cuestiones haya sido objeto de un amplio debate, ni tampoco que existiera una demanda de solución más o menos generalizado en el ámbito universitario europeo. Por eso, aunque tales cuestiones se hayan enfatizado una y otra vez, creo que es más adecuado entender Bolonia como un proyecto para "la institucionalización de la Educación Superior como un área o espacio europeo" y complementariamente la utilización por parte de los gobiernos "de una dimensión institucional europea como medio de legitimar reformas"10. Un proyecto legítimo que pretende ser una respuesta a un mercado cada vez más competitivo que requiere personas con mayores capacidades y competencias profesionales.

Supuesto lo anterior, lo que en apariencia se presenta como los focos fundamentales de preocupación que están en la base de Bolonia, podrían ser más bien cuestiones de segundo orden, sobre todo si se tiene en cuenta el tipo de soluciones que se han ido planteando. Así, como señala Carabaña, "la obvia inadecuación entre fines y medios manifiestos ha inducido a muchos a pensar que se trata de una tapadera bajo la cual se esconden agendas que son la verdadera razón del proceso"11.

Algunas pistas sobre esas otras razones del proceso podemos encontrarlas en la Declaración de la Comisión Europea de $2003^{12}$ en la que se señala claramente la necesidad de mejorar la cooperación entre las universidades y la empresa y también de reorganizar los conocimientos, y de responder a nuevas necesidades En este último sentido se afirma lo siguiente: "La universidad debe responder a las nuevas necesidades en materia de educación y formación que surgen con la economía y la sociedad del conocimiento y, especialmente, la necesidad cada vez mayor de enseñanza científica y técnica, de competencias transversales y de posibilidades de aprendizaje permanente". Lo que parece obvio es que la universidad está llamada a jugar un nuevo papel en la sociedad del conocimiento, un papel en el que la empleabilidad ocupa un lugar central.

De tal manera que lo que Bolonia persigue va mucho más allá de la búsqueda de la homogeneidad organizativa, el fomento de la movilidad o el desarrollo de los sistemas de calidad. Como afirma López Herrerias, "cambiamos la universidad, lo hacemos mediante la gran mediación del Espacio Europeo de Educación Superior, en nombre del crecimiento social y humano y de la ciudadanía europea, para facilitar la ocupabilidad"13.

Tomando en consideración todo lo anterior, no parece exagerado afirmar que Bolonia es más bien fruto de la demanda del mercado que de la de la propia sociedad, y menos aún todavía la de la universidad europea. Bolonia se asemeja más al diseño de un instrumento con capacidad de ir modificando el modus operandi de las universidades que a la respuesta de un problema planteado por los universitarios. Bolonia es un programa de acción más bien invisible que incorpora, a la vez y de manera visible, la justificación de ese programa.

Merece la pena citar aquí la valoración del Círculo de Empresarios que, tras reafirmar la necesaria posición de la universidad al servicio de la sociedad, valora las propuestas de la Comisión Europea: "En nuestro entorno inmediato encontramos un reconocimiento de esa realidad en las propuestas de la Comisión Europea. Así, se plantea la necesidad de modernizar la Universidad para flexibilizarla y adaptarla a los nuevos desafios, incrementando la competitividad de la economía europea. En esa línea, la Comisión propone varias líneas de actuación, como la eliminación de barreras que restan movilidad, la autonomía y responsabilidad de los centros, una mayor asociación con el mundo de la empresa, así como una mejor capacidad de ofrecer competencias y conocimientos adecuados para el mercado laboral (...). Se trata de integrar a la Universidad de manera más decidida en el tejido económico y productivo, fomentando la excelencia a través de la competencia"14.

\subsection{Los actores de Bolonia}

Ha escrito Innerarity: "Hace tiempo que las innovaciones no proceden de instancias políticas sino de la inventiva que se agudiza en otros espacios de la sociedad"15. Bolonia es un 
proceso planteado y diseñado desde la política, no desde la universidad. Es cierto que tiene cono antecedente la Magna Charta Universitatum suscrita por 388 rectores en 1988. Pero no lo es menos que las declaraciones de la Sorbona y Bolonia son ya cosa de los políticos. Como bien indica Del Valle, "las necesidades funcionales del mercado y del espacio público europeo, unidas a las transformaciones en la transmisión del conocimiento y los nuevos planteamientos de la globalización, justifican que el proceso del EEES se fundamente en una fuerte voluntad política y que esté implantándose en los Estados aún sin un marco normativo internacional o de derecho UE" ${ }^{16}$. ¿Constituye esta dimensión política un problema? En mi opinión, en este caso sí.

Es cierto que cualquier proceso de estas características puede articularse de distintas maneras. Parto también del supuesto de que estos procesos rara vez suscitan unanimidades y son capaces de imponer su criterio a toda la colectividad. Por tanto, siempre deben considerarse las relaciones entre los actores, los desafíos que a cada uno de ellos se le presentan y los prejuicios y resistencias que pueden suscitarse. Pero todo ello mucho tiene que ver con los impulsores y los actores de cada proceso. En el caso concreto de Bolonia hay preguntas cuya respuesta puede ayudarnos a clarificar ciertas dudas: ¿Es posible innovar en un ámbito en el que muchos de los actores fundamentales ni comparten los objetivos y medios que se plantean, ni muestran una disposición favorable hacia ellos? ¿Puede augurarse exitoso un proceso que nace bastante desvinculado del sentir de una buena parte de la comunidad universitaria? ¿Cuál es el sentir en la universidad española?

Creo que es bastante certera la descripción que hace López Heredia: "Predomina entre sus actores una salida individualista, cada uno a lo suyo, como manera de higienizarse en un contexto bastante ingrato para una amplia mayoría. (...) Domina un estilo de cierta latencia espiritual en el conjunto de las actividades de profesores y alumnos: clases, apuntes, propuestas de exámenes y algún aspecto más, añadible"17. Aunque esta perspectiva pueda matizarse, lo que resulta difícilmente discutible es la sensación de un profesorado expectante cuando no ausente, y un alumnado escéptico y desinformado, sólo ocasional y minoritariamente combativo.

Lo paradójico es que en el tiempo de las personas, cuando la mayoría de los discursos innovadores ponen el acento en la necesidad de contar con las personas, fomentando la creatividad, la participación y los proyectos comunes, Bolonia se percibe como un proyecto distante, bastante desconocido, con un gran componente técnico y escasamente compartido por una parte de los actores universitarios. Tal vez, la siguiente reflexión de Villa contenga alguna de las claves del distanciamiento que se percibe en sectores de la universidad española. Dice Villa: "en esta reforma que pretende adaptar la universidad al EEES en ningún momento se destaca como finalidad la formación de las personas, de los profesionales o de los científicos; sino el número de créditos, el cambio de metodología, la estructura o la calidad educativa. (...) Si las universidades se empeñan únicamente en cuidar y programar la metodología, la estructura y la calidad de sus titulaciones, dejarán en el olvido otras dimensiones que son necesarias para ser profesionales de calidad técnica y humana"18.

La universidad tiene su propia forma de hablar y también su particular visión de la historia y del papel social que le corresponde desarrollar, en definitiva su cultura. Tal cultura ni ha sido, ni es, ni puede ser inmutable. Pero tampoco se pueden pasar por alto los efectos que determinadas propuestas pueden tener para los miembros del colectivo universitario, para su cultura. La medida en que los actores universitarios vayan a comprometerse con Bolonia depende también del grado de conformidad con el proceso. Bolonia es un proceso que se ha planteado de arriba hacia abajo, que se ha discutido poco, pero se ha explicado menos. Esta es una primera y severa limitación. Bolonia es además una propuesta emanada desde la política, pero son los universitarios los que han de ponerla en práctica. Segunda y no menos importante limitación. Se trata de dos barreras que deberá superar un proceso en el que muchos de los actores principales no han participado. Aunque siempre cabe encontrar soluciones como las que plantea Gil Calvo: " $¿ A l-$ guien puede pensar que las nuevas ordenanzas burocráticas de Bolonia lograrán arreglar las cosas? Habrá que esperar y ver, para poder creerlo. Pero lo que sí parece seguro es que, de acuerdo a nuestra tradición (hecha la ley, hecha la trampa), una y otra parte, profesores y alumnos, aprenderemos a adaptarnos al embolado de Bolonia"19.

\section{3. ¿Quién lidera Bolonia?}

Una de las cuestiones más sorprendentes en relación al desarrollo del Proceso de Bolonia en la universidad española 
es la falta de un liderazgo activo y reconocible, capaz de concitar adhesiones y poner en valor el proyecto. Lo que en mi opinión debe entenderse como ausencia de liderazgo suscita también algunas preguntas: ¿Puede pensarse que es suficiente para liderar un proceso de estas caracteristicas un conjunto de declaraciones públicas bianuales, planteadas desde el ámbito europeo?; ¿Se ha logrado a través de tales declaraciones explicar adecuadamente los objetivos y los fines de Bolonia?; ¿A quién correspondia realmente liderar el proceso? Respecto a la última cuestión parece obvio que los escenarios eran básicamente dos: liderazgo político o liderazgo universitario. A mi entender, ni uno ni otro se han producido.

En primer lugar y en relación al ámbito político, puede afirmarse con rotundidad que, en el caso de España, los sucesivos gobiernos han estado desaparecidos. Una posible razón es la falta de continuidad de los responsables educativos. De hecho, entre 1999 y 2010 ha habido hasta seis ministros de educación distintos. Otra razón, bastante verosímil también, es una cierta indefinición sobre el papel que correspondía a los gobiernos y a las universidades en este proceso. Como indica Villa, "la declaración de Bolonia se fija en universidades, no en países. Sin embargo, como muy bien decía Ortega y Gasset, las diferencias las marcan los paises y sus peculiaridades propias"20. Una tercera razón puede tener que ver con la idea, también orteguiana de que Europa es la solución. Algo así parece deducirse de la opción tomada por las autoridades educativas españolas de optar por una adaptación drástica ${ }^{21}$ al EEES. El carácter europeo de Bolonia sería la propia razón legitimadora y haría innecesarios liderazgos más activos.

En segundo lugar, y por lo que respecta al ámbito universitario, la Asamblea General de la Conferencia de Rectores de las Universidades Españolas ha emitido cuatro Declaraciones y un Informe ${ }^{22}$ desde la Declaración de Bolonia de 1999. Un análisis detallado de dichos documentos ofrece tres conclusiones fundamentales:

- La CRUE ha sido, desde su origen, manifiestamente favorable a los principios enunciados en la Declaración de Bolonia y al proceso y los objetivos de construcción de un Espacio Europeo de Educación Superior.

- La CRUE ha participado de manera activa junto con el Ministerio de Educación en el diseño de la estructura normativa (Decretos, Órdenes, etc.) que ha regulado la adecuación de las universidades españolas a los requisitos planteados por Bolonia.

- La CRUE ha reiterado en sus declaraciones la necesidad de una financiación adicional para el proceso de convergencia europea. En el último Informe de septiembre de 2006 sobre la "Organización de las Enseñanzas Universitarias en España", en el punto 13. se dice literalmente: "Ia CRUE expresa inequívocamente al Ministerio de Educación y Ciencia que resulta imprescindible disponer de una financiación 'adicional y específica' para el desarrollo del proceso de convergencia europea".

Más allá de estos documentos no puede decirse que haya existido una tarea informativa y de divulgación dirigida a la comunidad universitaria. Desde la universidad, Bolonia se ha vivido más bien como algo que va a llegar o suceder tarde o temprano. En cierto sentido algo irremediable y también externo, para muchos impuesto.

La realidad es que se ha carecido de un liderazgo reconocible y mucho menos de iniciativas sostenidas que motivaran a la comunidad universitaria a participar. Bolonia ha sido al final un imponderable con fecha de implantación, un proceso de delegación en cascada desde la Comisión Europea a los Gobiernos, de estos a los Ministerios, de los Ministerios a los Rectores, de los Rectores a los Decanos, y de estos últimos al resto de la comunidad universitaria. Es más bien la historia de una urgente e ingente labor administrativa carente de estímulo y de entusiasmo.

\section{2. ¿SE InNOVA Aplicando Bolonia?}

Hay propuestas aparentemente atractivas, que se presentan como novedosas y capaces de cambiar sustancialmente determinados escenarios, pero que cuando se analizan en detalle nos damos cuenta de que buena parte de su contenido es ya conocido e incluso está aplicado y sus potencialidades son bastantes más modestas de lo que sus promotores pretenden sugerir.

Algo de esto sucede con Bolonia, una propuesta que analizada en detalle no resulta tan potencialmente transformadora y su textura real es bastante distinta. Quiero repasar 
aquí algunas de sus prioridades implícitas, interpretadas como transformaciones que han quedado ya asociadas a este proceso.

\subsection{A vueltas con la movilidad}

Probablemente la puesta en marcha del Programa Erasmus ha constituido el mejor y mayor impulso para la movilidad de los estudiantes en el ámbito europeo. Su inicio data del año 1987 y más de dos décadas más tarde puede decirse que un porcentaje significativo de los estudiantes europeos cursan alguno de sus años universitarios en una universidad y un pais distintos al propio. Para posibilitar el desarrollo de Erasmus las universidades han desarrollado múltiples acuerdos de cooperación bilateral o multilateral a través de los cuales se produce de facto el reconocimiento entre unas y otras. Bien es cierto que Erasmus es un programa limitado, pues tiene por objeto la movilidad del alumnado.

Los objetivos de Bolonia son en este terreno más ambiciosos. Lo que se declara perseguir es no sólo eliminar cualquier obstáculo a la movilidad del conjunto del personal universitario, sino también de titulados y profesionales en el ámbito de la Unión Europea. Para ello se plantean intervenciones tales como la unificación del sistema de títulos, la introducción del crédito ECTS o el suplemento europeo al título. Sin embargo, el resultado de tales intervenciones es más que dudoso.

Por lo que se refiere a la unificación del sistema de títulos la conclusión en el caso español no puede ser más sorprendente. La nueva estructura se basa, obviamente, en dos ciclos principales (grado y postgrado), pero reduce los cinco años necesarios para obtener las antiguas licenciaturas a cuatro años en lo que respecta a los nuevos grados. Lo sorprendente es que en la mayoría de las universidades europeas la duración de los grados será de tres años, de tal suerte que un estudiante que curse sus estudios de grado en una universidad española deberá cursar un año más que sus homólogos europeos para obtener el mismo reconocimiento académico. Por lo tanto, la ansiada equivalencia se produce de una manera ciertamente atípica reconociendo como equivalentes grados de distinta duración. Por cierto, algo que puede tener pésimas consecuencias.

Aunque no fuera fácil de entender, podría haberse restado importancia a lo anterior si se hubiera producido paralela- mente una unificación de contenidos y curricula. Nada más lejos de la realidad. Variadas razones (distintas tradiciones universitarias, denominaciones, contenidos, etc.) explican que la pretendida equivalencia de contenidos se haya convertido en una simple declaración de intenciones ${ }^{23}$.

De tal manera que la equivalencia no puede sino basarse en un sistema de reconocimiento que ya existía previamente y mejorarse, en todo caso, a través de la formulación de un conjunto de competencias presentes entre los objetivos a desarrollar en cada titulación. Como bien indica Carabaña, "en realidad, la única política eficaz contra el obstáculo que suponen los largos procesos de homologación de títulos es el reconocimiento automático por todos los Estados de los títulos avalados por cada uno de ellos, quizás tras acuerdos sobre su duración y contenidos mínimos"24.

El crédito ECTS (European Credit Transfer and Accumulation System), es otra de las herramientas Ilamadas a facilitar la movilidad en el ámbito universitario europeo. Como explica López Herrerías, "que es un sistema de 'transferencia de créditos' conecta con que facilita el reconocimiento de períodos de estudio en el extranjero. Que es un sistema de 'acumulación' tiene que ver con que computa créditos realizados en diferentes contextos, ya institucional, regional, nacional o europeo"25. La cuestión del reconocimiento conecta directamente con la movilidad. La de la acumulación, es una vía no expresada de manera explícita, para asimilar procedimientos y metodologías en los procesos de enseñanza/aprendizaje. Por tanto, es sobre todo la cuestión del reconocimiento la que realmente puede simplificar y favorecer la movilidad.

Queda, por fin, el suplemento europeo al título que no es algo distinto a un formulario bastante extenso, eso sí unificado para el conjunto de las universidades europeas en el que se refleja bastante información de la trayectoria de cada estudiante a lo largo de sus estudios. Como bien observa Carabaña, "nada nuevo, aunque sí eficaz. (...) Lo que resulta un tanto sospechoso es que se incorpore de oficio a unos títulos que se prometen fácilmente reconocibles e intercambiables"26.

Mi impresión es que bajo la apariencia de un conjunto de medidas aparentemente novedosas y decididamente favorecedoras de la movilidad, la realidad confirma o refuerza la idea de que muchas de ellas no mejoran los soportes 
sobre los que la movilidad va a desarrollarse, sino que sanciona por la vía de los hechos determinadas prácticas que serán posibles fundamentalmente por la voluntad política que irá poco a poco transfiriéndose a la esfera jurídica.

\subsection{Los cambios metodológicos}

Otra de las grandes paradojas de este proceso es que lo que se ha venido en denominar la innovación en los sistemas de enseñanza/aprendizaje, algo que no sólo afectaría finalmente a la manera en que los estudiantes aprenden, sino también a lo que aprenden. Lo curioso, como bien recuerda Del Pozo es que: "el cambio de metodología en la enseñanza universitaria no fue uno de los objetivos iniciales que propulsaron el plan de Bolonia, aunque si ha resultado ser una de sus consecuencias. Una decisión académica, la implantación de los créditos ECTS, y otra económica, la incorporación de habilidades y competencias que conecten a los estudiantes con el mercado laboral, se delinean como los dos factores que han propiciado la renovación metodológica. Muy progresivamente se ha ido construyendo el concepto de 'educación superior centrada en el alumno'"27.

Esta nueva orientación resulta además más comprometida con el saber/hacer y el saber/ser que con el saber a secas. Se advierte una mayor preocupación por los procedimientos, los sistemas de evaluación, o un seguimiento más integral de la actividad de los estudiantes ${ }^{28}$. En palabras de Rebollo, "la formación universitaria, que durante largo tiempo se ha basado en la transmisión de saberes, se plantea en la actualidad como el desarrollo y adquisición de competencias profesionales" 29 . Algo que concuerda, por otra parte, con la nueva manera de entender la dedicación de los estudiantes asociada al ECTS. Sin embargo, da la impresión de que en torno a esta cuestión no sólo se ha tratado de producir un consenso que no existe, sino también de tildar de novedosos procedimientos de uso bastante ordinario en nuestras universidades.

Con respecto a la primera cuestión existen muchas divergencias entre el profesorado universitario en torno a los fines y objetivos de las diferentes materias, la importancia de los sistemas de aprendizaje, o si las nuevas propuestas de seguimiento y evaluación son coherentes con los objetivos definidos. Es más, la idea de una educación superior centrada en el alumno ha de ser considerada con prudencia. Porque el buen rendimiento y los resultados de una universidad están todavía íntimamente ligados a la manera en que se configura y se desarrolla el tiempo de enseñanza/ aprendizaje. En tal sentido, en el ámbito universitario no existen evidencias suficientes que demuestren la supremacía de determinadas metodologías. Creer que un cambio severo de los procedimientos metodológicos producirá una mejora de los resultados es sencillamente una ingenuidad. La experiencia demuestra que ello está lejos de ser fácil y que es conveniente desconfiar de discursos pedagogistas que tienden a otorgar carácter de totalidad a determinadas actividades que son sólo parcialmente satisfactorias.

Además, es bien sabido que el aprendizaje de un mismo saber, saber/hacer o saber/ser puede efectuarse siguiendo muchas vías y muy diferentes modalidades. Las ventajas relativas de unas y otras así como sus eventuales consecuencias son también objeto de discusión. No es buen camino razonar sobre lo absoluto. Determinados cambios en los procedimientos suscitan problemas que muchos profesores no están en condiciones de afrontar. En mi opinión, el análisis sobre estas cuestiones debería tener muy en cuenta la siguiente afirmación de Zabalza: "Lo que la Universidad y los profesores universitarios podemos dar a nuestros estudiantes es ese plus de aprendizaje y desarrollo formativo que ellos no podrían adquirir por sí solos. (...) Con nosotros deberían poder hacer cosas que no podrían hacer, harían peor o tardarían más en lograr por ellos mismos. (...) Eso debería ser, en definitiva, la docencia de calidad"30.

En cuanto al carácter novedoso de muchas de las modalidades docentes que ciertos profesionales asocian a Bolonia la experiencia demuestra que ello está bastante alejado de la realidad. En la universidad, muchos profesores Ilevan años experimentando diversos procedimientos formativos y construyendo sus propios instrumentos, ambas consideradas actividades inherentes a su desempeño profesional. Como bien subraya López Herrerías "hay prácticas, hay tutorías, hay trabajo en grupo, hay proyectos. ¡Bueno, se puede insistir en la propuesta de animar una variedad de actividades de aprendizaje! Eso sí, no presentarlo como una novedad tajante de lo que se hace" ${ }^{\text {"31. }}$.

Creo sinceramente que muchos programas y discursos pedagogistas han versado más sobre las intenciones, sobre lo que se quiere que aprendan los estudiantes y sobre cómo se quiere que lo aprendan, que sobre los niveles efectivos de aprendizaje que deben asegurarse (por curso, ciclo, etc.) 
Y por lo general, muchos de estos discursos carecen de un mínimo diagnóstico previo.

No hay tanta innovación como la que se predica y asocia a la generalización de más modalidades de enseñanza/ aprendizaje. Algunas de ellas ya son aplicadas por una mayoría del profesorado, otras están casi estandarizadas, otras por fin deberán dar cuenta de sus capacidades y potencialidades innovadoras. La universidad hace tiempo que ha diversificado y ampliado sus procedimientos metodológicos. Lo sorprendente es la escasez de informaciones precisas y seguras que disponemos sobre las bondades de estos procedimientos, y su valor añadido en comparación con otro tipo de prácticas docentes de corte más clásico.

\subsection{La cultura de la evaluación}

Una de las cuestiones más novedosas que cabe asociar a Bolonia es el desarrollo de un nuevo modelo de evaluación y supervisión de las universidades. Se trata de que este nuevo modelo, edificado sobre la cultura de la calidad, quede inserto entre los objetivos y metas generales de las universidades y abarque al conjunto de actores y actividades que se desarrollan en la universidad. De una parte, el ámbito de académico/formativo que involucra a distintos actores (profesorado, alumnado, etc.) y también diversas actividades (procesos de enseñanza/aprendizaje, investigación...). De la otra, el ámbito de la actividad gestora y administrativa (servicios, medios infraestructurales y financieros, etc.).

La clave de este sistema de garantía de calidad es la acreditación que no es sino un mecanismo de control y reconocimiento de la actividad de las universidades. En cierto sentido, Bolonia acelera la introducción de estas prácticas en las universidades al vincular la garantía de calidad con el reconocimiento de títulos. Sobre la base de este requerimiento inicial en casi todos los estados se han ido desarrollando sistemas de acreditación que, por lo general, los pone en marcha el gobierno y sirven para ejercer sus funciones de control de calidad.

Lógicamente lo anterior ha de implicar la determinación de criterios o estándares que ayuden a edificar un marco de certificación de la calidad que sea reconocible y comparable. Como bien se ha señalado, "la acreditación debe ser considerada como el método para evaluar la calidad y la pertinencia de una institución o programa determinado de educación superior, permitiendo que sea reconocida dentro de un sistema de educación superior existente (especificado)"32.

En el caso español, la ANECA (Agencia Nacional de Evaluación de la Calidad y Acreditación) junto a una docena de agencias autonómicas vienen definiendo los objetivos básicos de su actividad de evaluación institucional que pueden sintetizarse en dos $^{33}$ :

- Constituirse en herramienta para ayudar a las titulaciones 0 instituciones a gestionar y mejorar la calidad de la formación universitaria, es decir, para ayudar a producir cambios significativos en el diseño de las titulaciones y en el proceso de enseñanza-aprendizaje.

- Facilitar que se dé mayor satisfacción a las demandas de formación de los estudiantes y mejor respuesta a las demandas sociales, garantizando la eficacia y eficiencia de las inversiones en enseñanza superior, así como mejorar la calidad de la información que se ofrece a la sociedad sobre el funcionamiento de su sistema de educación superior.

El seguimiento y evaluación externa de las titulaciones y por ende de la actividad de las universidades se convierte entonces en un requisito que adquiere rango legal. Así, "la autonomía en el diseño del título se combina con un adecuado sistema de evaluación y acreditación, que permitirá supervisar la ejecución efectiva de las enseñanzas e informar a la sociedad sobre la calidad de las mismas" ${ }^{\prime \prime 4}$. Pero la cultura de la evaluación no se limita al ámbito de las titulaciones sino que afecta a la actividad del personal docente e investigador, la actividad de gestión del profesorado, los sistemas de promoción profesional, etc.

En los últimos años, la cultura de la calidad ha ido penetrando en la universidad española, invadiendo cada vez más espacios de actuación y gestión. Desde mi perspectiva, esta penetración de la cultura de la calidad se ha debido fundamentalmente a la iniciativa externa que ha ido obligando a la universidad al desarrollo de procedimientos, procesos, protocolos, controles, seguimientos, etc., con resultados de momento poco conocidos. Ni que decir tiene que las opiniones son diversas y que la idoneidad de muchos de los estándares de calidad definidos es muy discutible y discutida. También resulta evidente que la cultura de la 
calidad ha multiplicado la burocracia y desatado el furor credencialista en gran parte del profesorado universitario.

En definitiva, de una parte, la determinación de los estados en crear un sistema comparable de acreditación internacional es firme y aunque todavía estamos lejos de poseer un lenguaje común para la acreditación y la garantía de calidad, mi impresión es que el camino emprendido no tiene retorno. Como ha señalado Ginés Mora, "el desarrollo de experiencias nacionales llevadas a cabo con acuerdos mutuos entre paises con sistemas relativamente similares permitirá el desarrollo de un consenso en los procedimientos que podría llevar en un plazo relativamente corto al reconocimiento mutuo de las acreditaciones y, quizá, en un plazo más largo, a la existencia de una acreditación europea única" ${ }^{\prime 3}$.

Por otra parte, y en contraste con lo anterior, la universidad española ha adoptado, una vez más, el papel de observador/receptor/cumplidor de las iniciativas externas, y en absoluto de actor principal de estos procesos. Mientras tanto, un ejército de evaluadores de muy diverso signo, condición y cometido ocupa ya un lugar preferente y estratégico en nuestro sistema de educación superior, un indicador inequívoco de que la cultura de la calidad no es una moda pasajera sino que ha llegado para quedarse.

\section{Conclusiones}

Han escrito Hargreaves y Fink: "El cambio en la educación es fácil de proponer, complicado de llevar a la práctica y extraordinariamente difícil de sostener. (...) Las innovaciones atraen pronto a los primeros entusiastas, pero más difícil es convencer a los educadores más escépticos para que se unan a la labor de hacer realidad el cambio" ${ }^{36}$. Lo que se ha conocido como el "Proceso de Bolonia" participa plenamente, en mi opinión, de los problemas descritos en el párrafo anterior. Es más, mientras muchos de los universitarios siguen discutiendo sobre la pertinencia de lo que se ha hecho y lo que se debe hacer o no hacer, las decisiones ya están tomadas y se están poniendo en marcha.

Mientras Bolonia se ha planteado como la hoja de ruta para la innovación de la Enseñanza Superior en Europa la universidad española, lejos de adoptar una estrategia proactiva que no acritica, se ha conformado con ir acep- tando sus efectos desde una alarmante pasividad, en algunas ocasiones mezclada con actitudes reactivas. Este hecho puede estar teniendo ya consecuencias que podrán incrementarse en el futuro, porque como bien advierte Gurrutxaga, "los caminos de la innovación son diversos, algunos implican transformaciones de ruptura, otros innovan en escalas de menos alcance. No participar en el club de los primeros no impide participar en el club de los segundos, el problema es estar fuera de ambos" ${ }^{\prime 37}$.

La universidad, las universidades no pueden obviar un escenario internacional marcado por la competitividad y el peso de la globalización. No pueden tampoco ignorar, no digo que deban desearlo, el peso y la influencia creciente del mercado. Ni deben dar la espalda al cuestionamiento de ciertas formas de hacer y reportar a la sociedad de las instituciones universitarias. Las universidades han de saber que no pueden estar ausentes de los procesos innovadores, que han de ser capaces de formular alternativas, de edificar propuestas propias. La universidad no puede limitarse a asumir pasivamente los discursos que provienen del exterior, no pueden convertir las orientaciones en dogmas, debe edificar su propio discurso.

Bolonia se ha presentado como la propuesta innovadora de la Educación Superior en Europa. He tratado de mostrar que Bolonia no es, sino parcialmente, un proceso realmente innovador y que algunas de sus propuestas son ya realidades en el funcionamiento ordinario de las universidades. Pero Bolonia si era y es una oportunidad para la reflexión, el contraste, el debate, la generación de nuevas ideas, es decir, lo que se entiende como la antesala de la innovación. Si hoy aparece a los ojos de muchos como un proceso impuesto, ello se debe en buena medida a la actitud de nuestras universidades.

Creo que Bolonia es la historia de un proceso apenas liderado, cuando menos de manera visible, muy poco y mal explicado y escasamente discutido. Pero ello no justifica la pasividad de los universitarios y de las universidades españolas. No justifica que desde la universidad no se hayan rebatido y explicado que muchos de los cambios emprendidos no eran absolutamente necesario ${ }^{38} ;$ que la universidad haya en cierto sentido delegado su responsabilidad en la determinación de lo que constituye formar ciudadanos y no sólo profesionales; que no tomemos partido en torno a lo que significa la calidad o la excelencia como horizonte 
de nuestra actividad; que no hayamos advertido de que cambiar los sistemas universitarios en apenas una década puede ser demasiado precipitado.

Lo paradójico es que Bolonia es ahora la ruta, y la lógica pregunta es qué hacemos si ya la estamos transitando. Demasiado estrés, demasiadas prisas y poca reflexividad. La innovación poco tiene que ver con la improvisación, salvo que queramos que el cambio no sea a mejor sino a peor. Entiendo que la innovación no se edifica sobre las ruinas del pasado sino que aprende de él. El saber lento, las formas lentas de conocer, no son contradictorias con las actitudes innovadoras. Bolonia ya avanza y por eso ahora más que nunca cada universidad está impelida a definir su futuro. $Y$ para ello nada mejor que establecer, cuando menos, los objetivos que se persiguen y el lugar que se pretende ocupar.

En este último sentido, a cada universidad corresponde ir definiendo como imagina ese lugar en lo que ya es un mercado cada vez más exigente e internacionalizado. Y en el caso de que el objetivo sea la excelencia y aunque no existan fórmulas mágicas, sí que conocemos los requerimientos que debemos satisfacer: "Las universidades de mayor categoría son las que hacen importantes contribuciones al progreso del conocimiento mediante la investigación, las que enseñan con los programas de estudios y los métodos pedagógicos más innovadores, bajo las condiciones más propicias, las que hacen de la investigación un componente integral de la enseñanza de pregrado y las que producen profesionales que se destacan debido a su éxito en ámbitos altamente competitivos durante su educación, y (lo que es más importante) después de su graduación. Son estos logros concretos y la reputación internacional asociada a éstos los que hacen que estas instituciones sean consideradas de rango mundial" ${ }^{\prime 2}$. Entre la cima de la excelencia y la más vulgar de las mediocridades existen muchos intervalos. Pero hay que elegir un destino antes de que otros nos lo señalen.

\section{NOTAS}

1 Sobre el origen, evolución y retos de la evolución superior puede consultarse Calderón, R. (2009): "El nuevo papel de la Educación Superior", en García Manjón, J. V. (coord.): Hacia el Espacio Europeo de Educación Superior, La Coruña, Netbiblo, pp. 22-41; UNESCO (1998): La Educación Superior en el siglo XXI: visión y acción, Paris, Unesco; Salaburu, P. (2007): La Universidad en la encrucijada. Europa y Estados Unidos, Madrid, Academia Europea de Ciencias y Artes.

2 Brunner, J. J. (2009): "La universidad, sus derechos e incierto futuro", en Revista Iberoamericana de Educación, n. ${ }^{4}$ 49, pp. 77-102, p. 92.

3 El texto de esta declaración contiene un preámbulo, cuatro principios fundamentales, y un conjunto de medios para llevar a cabo tales principios. Ratifica la autonomía de la universidad, la simbiosis entre las actividades docente e investigadora, el carácter universal del saber, y la importancia de desarrollar iniciativas comunes entre las universidades europeas, lo que se expresa literalmente como sigue: "las universidades alientan la movilidad de los profesores y de los estudiantes, y estiman que una política general de equivalencia en materia de estatutos, de títulos, de exámenes (aún manteniendo diplomas nacionales) y de concesión de becas, constituye el instrumento esencial para garantizar el ejercicio de su misión contemporánea".

4 Esta declaración, firmada por los ministros de educación de Francia, Reino Unido, Italia y Alemania, además de referirse ya explicitamente a un área europea de Educación Superior, se refiere ya a la existencia de dos ciclos, menciona el sistema de créditos ECTS, a la movilidad, y a la necesidad de lograr una armonización progresiva de las titulaciones.
Recibido: 27 de julio de 2010

Aceptado: 31 de octubre de 2010 
5 La Declaración de Bolonia fue firmada por representantes educativos de 28 países y se entiende como la hoja de ruta para la Educación Superior en el ámbito europeo.

6 Fernández Buey, F. (2009): Por una universidad democrática, Barcelona, El Viejo Topo, p. 298.

7 Ver a este respecto Gurrutxaga, A. (2009): "Recorridos por la Innovación", en Innerarity, D. y Gurrutxaga, A., ¿Cómo es una sociedad innovadora?, Zamudio, Innobasque.

8 Ver Echeverría, J. (2010): "Ocho Propuestas sobre Innovación Social", en Gurrutxaga, A. y Echeverria, J., La luz de la luciérnaga. Diálogos de Innovación Social, Zarautz, ASCIDE, pp. 72-74.

9 Ver, por ejemplo, Chesbrough, H. W. (2009): Innovación Abierta, Barcelona, Ed. Plataforma; Gurrutxaga, A. (2010): Recorridos por el cambio, la innovación y la incertidumbre, Bilbao, UPV-EHU.; Ibarra, A.; Castro, J., y Rocca, L. (2006): Las Ciencias Sociales y las Humanidades en los Sistemas de Innovación, Bilbao, UPV-EHU; Florida, R. (2010): La Clase Creativa, Barcelona, Paidós.

10 Ver Luzón, A.; Sevilla, D. y Torres, M. (2009): "El proceso de Bolonia: significado, objetivos y controversias", en Avances en Supervisión Educativa n. 10 , abril.

11 Carabaña, J. (2006): "Las agendas ocultas tras Bolonia", en Crítica, n. 934 , pp. 36-41, p. 38.

12 Comisión Europea (2003): El papel de las universidades en la Europa del conocimiento, Bruselas, Comisión de las Comunidades Europeas.

13 López Herrerias, J. A. (2008): La Universidad (y el proceso de Bolonia: EEES), Huelva, Hergué Impresores, p. 102.

14 Círculo de Empresarios (2007): Una Universidad al servicio de la socie- dad, Madrid, Círculo de Empresarios, pp. 5-6.

15 Innerarity, D. (2009): "La sociedad de la Innovación", en Innerarity, D. y Gurrutxaga, A., ¿Cómo es una sociedad innovadora), ob. cit., p. 23.

16 Del Valle Gálvez, A. (2006): "El espacio europeo de educación superior -planteamiento y perspectivas-", en Revista Electrónica de Estudios Internacionales, n. ${ }^{\circ} 11$, Madrid, Asociación Española de Profesores de Derecho Internacional y Relaciones Internacionales.

17 López Herrerias, J. A. (2008): La Universidad (y el proceso de Bolonia: EEES), ob. cit., p. 137.

18 Villa, J. (2008): "La Misión de la Universidad. Antes y después de una misma tarea", en Flecha, J. R. (coord.): El proceso de Bolonia y la Enseñanza Superior en Europa, Salamanca, Universidad Pontificia de Salamanca, Comunicaciones, pp. 33-48 y pp. $44,47$.

19 Gil Calvo, E. (2009): "A propósito de Bolonia", artículo publicado en el Diario El País, 13-05-2009.

20 Villa, J. (2008): La Misión de la Universidad. Antes y después de una misma tarea, ob. cit., p. 47.

21 Ver Carabaña, J. (2006): "Bolonia: ¿otro espejismo europeo?", en Cuadernos de Información Económica, Madrid, Fundación de las Cajas de Ahorros n. ${ }^{\circ} 190$, enero-febrero, pp. 163-172.

22 Concretamente en los años 2000, 20002, 2003 (septiembre), 2003 (octubre) y 2006.

23 Más allá de la excepción en el caso de los estudios de Medicina.

24 Carabaña, J. (2006): "Las agendas ocultas tras Bolonia", en Crítica, n. ${ }^{\circ}$ 934, pp. 36-41 y p. 38.

25 López Herrerias, J. A. (2008): La Universidad (y el proceso de Bolonia: EEES), ob. cit., p. 131.
26 Carabaña, J. (2006): "Las agendas ocultas tras Bolonia", en Crítica, n. ${ }^{\circ}$ 934, pp. 36-41 y p. 37.

27 Del Pozo Andrés, M. M. (2008): "El proceso de Bolonia en las aulas universitarias: Una perspectiva europea", en Cuestiones Pedagógicas, n. 19, Sevilla, Secretariado de Publicaciones, Universidad de Sevilla, pp. 5573 y p. 63

28 La producción al respecto es ingente. A modo de referencia puede consultarse Van der Hofstadt, C. y Gómez, J. (2006): Competencias y Habilidades Profesionales para universitarios, Madrid, Díaz de Santos; Palomares, T.; González, M. N. y Madariaga, J. M. (eds.) (2008): La Innovación Educativa en la Universidad: adaptación al cambio, Bilbao, UPV/EHU; Imbernon, J. (2009): Mejora de la enseñanza y el aprendizaje en la universidad, Cuadernos de Docencia Universitaria 14 Barcelona, ICE-0ctaedro.

29 Rebollo, M. A. (2005): "Experiencias en la aplicación del crédito europeo", en Colas, P. y De Pablos, J. (coords.): La Universidad en la Unión Europea. El Espacio Europeo de Educación Superior y su impacto en la docencia, Málaga, Ediciones Aljibe, pp. 125153.

30 Zabalza, M. A. (2003): Competencias docentes del profesorado universitario. Calidad y desarrollo profesional, Madrid, Narcea, p. 215.

31 López Herrerias, J. A. (2008): La Universidad (y el proceso de Bolonia: EEES), ob. cit., p. 177.

32 Van Hinkel, H. y Rodrigues, M. A. (2009): "Retos institucionales y políticos de la acreditación en el ámbito internacional", en Global University Network for Innovation, La Educación Superior en tiempos de cambio. Nuevas dinámicas para la responsabilidad social, Madrid-Barcelona- 
México, Ediciones Mundi-Prensa, pp. $162-168$ y p. 163.

33 Aneca y otras (2009): Informe Sobre el estado de la evaluación externa de la calidad en las universidades españolas, 2008, Madrid. www.aneca.es.

34 Ver Real Decreto 1393/2007, de 29 de octubre, por el que se establece la ordenación de las enseñanzas universitarias oficiales.

35 Ginés Mora, J. (2002): "La acreditación de los estudios universitarios españolas en el contexto europeo", en Universidad de La Rioja: La LOU en marcha. ¿Marcha la LOU?, Jornadas de Gerencia Universitaria, Logroño, Universidad de La Rioja.

36 Hargreaves, A. y Fink, D. (2008): El liderazgo sostenible. Siete principios para el liderazgo en centros educativos innovadores, Madrid, Ediciones Morata, p. 15.

37 Gurrutxaga, A. (2011): "Diálogo con la Innovación", en Gurrutxaga, A. y Echeverría, J., La luz de la luciérnaga. Diálogos de Innovación Social, Madrid, Plaza y Valdés, p. 17.

38 Por ejemplo, la reforma de los planes de estudio.

39 Salmi, J. (2009): El desafío de crear universidades de rango mundial, Washington, Banco Mundial.

\section{BIBLIOGRAFÍA}

Aneca y otras (2009): Informe sobre el estado de la evaluación externa de la calidad en las universidades españolas, 2008, Madrid. www.aneca.es.

Brunner, J. J. (2009): La universidad, sus derechos e incierto futuro, En Revista Iberoamericana de Educación n. ${ }^{\circ} 49$.

Carabaña, J. (2006): Bolonia: ¿otro espejismo europeo? En Cuadernos de Información Económica, Madrid, Funda- ción de las Cajas de Ahorros n. 190, enero-febrero.

Carabaña, J. (2006): Las agendas ocultas tras Bolonia. En Crítica, n. 934.

Chesbrough, H. W. (2009): Innovación Abierta, Barcelona, Ed. Plataforma.

Círculo de Empresarios (2007): Una Universidad al servicio de la sociedad, Madrid, Círculo de Empresarios.

Colas, P. y De Pablos, J. (coords.) (2005): La Universidad en la Unión Europea. El Espacio Europeo de Educación Superior y su impacto en la docencia, Málaga, Ediciones Aljibe.

Comisión Europea (2003): El papel de las universidades en la Europa del conocimiento, Bruselas, Comisión de las Comunidades Europeas.

CRUE (2000): Declaración de Bolonia: Adaptación del Sistema Universitario Español a sus Directrices. www.crue.org.

CRUE (2002): La Declaración de Bolonia y su repercusión en la estructura de las titulaciones de España. www.crue.org.

CRUE (2003): Declaración de la Conferencia de Rectores de las Universidades Españolas sobre el Espacio Europeo de Educación Superior, septiembre www. crue.org.

CRUE (2003): Declaración de la Conferencia de Rectores de las Universidades Españolas sobre el Espacio Europeo de Educación Superior, octubre www. crue.org.

CRUE (2006): Informe de la Conferencia de Rectores de las Universidades Españolas sobre la propuesta del documento de trabajo de fecha 26 de septiembre de 2006 "La Organización de las Enseñanzas Universitarias en España" presentado por el Ministerio de Educación y Ciencia, www.crue.org.

Del Valle Gálvez, A. (2006): "El espacio europeo de educación superior -planteamiento y perspectivas-", en Revista Electrónica de Estudios Internacionales, n. ${ }^{\circ} 11$, Madrid, Asociación
Española de Profesores de Derecho Internacional y Relaciones Internacionales.

Del Pozo Andrés, M. M. (2008): "El proceso de Bolonia en las aulas universitarias: Una perspectiva europea", en Cuestiones Pedagógicas, n. ${ }^{\circ}$ 19, Sevilla, Secretariado de Publicaciones, Universidad de Sevilla.

Fernández Buey, F. (2009): Por una universidad democrática, Barcelona, El Viejo Topo.

Flecha, J. R. (coord.) (2008): El proceso de Bolonia y la Enseñanza Superior en Europa, Salamanca, Universidad Pontificia de Salamanca, Comunicaciones.

Florida, R. (2010): La Clase Creativa, Barcelona, Paidós.

Garcia Manjón, J. V. (coord.) (2009): Hacia el Espacio Europeo de Educación Superior, La Coruña, Netbiblo.

Gil Calvo, E. (2009): A propósito de Bolonia, artículo publicado en el Diario El País, 13-05-2009.

Global University Network for Innovation (2009): La Educación Superior en tiempos de cambio. Nuevas dinámicas para la responsabilidad social, Madrid-Barcelona-México, Ediciones Mundi-Prensa.

Gurrutxaga, A. (2010): Recorridos por el cambio, la innovación y la incertidumbre, Bilbao, UPV-EHU.

Gurrutxaga, A. y Echeverría, J. (2010): La luz de la luciérnaga. Diálogos de Innovación Social, Zarautz, ASCIDE.

Hargreaves, A. y Fink, D. (2008): El liderazgo sostenible. Siete principios para el liderazgo en centros educativos innovadores, Madrid, Ediciones Morata.

Ibarra, A.; Castro, J. y Rocca, L. (2006): Las Ciencias Sociales y las Humanidades en los Sistemas de Innovación, Bilbao, UPV-EHU.

Imbernon, J. (2009): "Mejora de la enseñanza y el aprendizaje en la universidad", 
Cuadernos de Docencia Universitaria, 14, Barcelona, ICE-Octaedro.

López Herrerías, J. A. (2008): La Universidad (y el proceso de Bolonia: EEES), Huelva, Hergué Impresores.

Luzón, A.; Sevilla, D. y Torres, M. (2009): "El proceso de Bolonia: significado, objetivos y controversias", en Avances en Supervisión Educativa, n. ${ }^{10}$ abril.

Palomares, T.; González, M. N. y Madariaga, J. M. (eds.) (2008): La Innovación Educativa en la Universidad: adaptación al cambio, Bilbao, UPV/EHU.
Salaburu, P. (2007): La Universidad en la encrucijada. Europa y Estados Unidos, Madrid, Academia Europea de Ciencias y Artes.

Salmi, J. (2009): El desafío de crear universidades de rango mundial, Washington, Banco Mundial.

UNESCO (1998): La Educación Superior en el siglo XXI: visión y acción, París, Unesco.

Universidad de La Rioja (2002): La LOU en marcha. ¿Marcha la LOU?, Jornadas de Gerencia Universitaria, Logroño, Universidad de La Rioja.
Valle, J. M. (2005): "El 'Proceso de Bolonia', ¿Punto de partida o línea de llegada? Historia de la política educativa en la Unión Europea en materia de Educación Superior", en Quaderns Digitals, n. ${ }^{\circ} 38$, Valencia.

Van der Hofstadt, C. y Gómez, J. (2006): Competencias y Habilidades Profesionales para universitarios, Madrid, Díaz de Santos.

Zabalza, M. A. (2003): Competencias docentes del profesorado universitario. Calidad y desarrollo profesional, Madrid, Narcea. 